EDITORIAL

\title{
Depression and disability in coronary patients: time to focus on quality of life as an end point
}

D C Haas

\section{Depression in acute coronary syndrome patients may have prognostic implications beyond mortality}

S ubstantial prospective, epidemiologic evidence now suggests that among patients admitted to the hospital for an acute coronary syndrome (ACS), those suffering comorbid depression are at significantly increased risk for future cardiovascular morbidity and mortality. ${ }^{1-4}$ This appears to be true even for patients with mild depressive symptoms that do not meet full clinical criteria for major depression. ${ }^{5}$ Given the remarkable prevalence of depressive symptoms among patients with coronary disease (approximately $20-30 \%$ ), the issue is far from trivial. ${ }^{6}$ However, why depression is a risk factor for poor prognosis is unclear. In many studies, depression is an independent predictor of poor outcomes, which has prompted several investigators to suggest that depression itself may be causally related to the poor prognosis observed in depressed ACS patients. ${ }^{7}$ Other studies have demonstrated that depression is no longer an independent predictor of outcomes when the severity of coronary disease is rigorously measured and adjusted for in the analyses. ${ }^{8}$ This has led some to conclude that depression is a marker of disease severity, rather than an independent poor prognostic factor. ${ }^{89}$ Adding to the complexity of this debate is that while there are several plausible mechanisms through which depression may mediate worse outcomes, none has been proven to be the link engendering adverse clinical end points. ${ }^{10}$ Moreover, it is unknown if treating depression in ACS patients will improve cardiovascular outcomes. Two large trials $^{11}{ }^{12}$ have examined this issue; only one ${ }^{12}$ was powered to detect a reduction in clinical events, and it was a negative study. Yet despite the controversies and uncertainties regarding depression and coronary disease, it is important to not lose focus regarding three concerning facts with respect to ACS patients: (1) depression is common; (2) causal or not, depression portends a worse cardiovascular prognosis; and (3) these patients have a comparatively poorer quality of life.

\section{DEPRESSION AND CO-MORBID MEDICAL ILLNESS}

Given the potential significance of fully elucidating the true relationship between depression and prognosis among ACS patients, it is perhaps not surprising that research in this area has targeted
Heart 2006;92:8-10. doi: 10.1136/hrt.2005.066035

depression as a potentially modifiable risk factor for cardiovascular outcomes-substantially fewer prospective studies have examined the impact of depression on quality of life in ACS patients. ${ }^{13}$ That patients' quality of life should be an outcome of interest for clinicians is self evident. Long established data demonstrate that depression worsens quality of life in general and particularly in the presence of co-morbid medical illness (including coronary disease). ${ }^{14}$ However, quality of life studies may be particularly meaningful within the depression-coronary disease debate, since it has been shown that self perceived cardiac health status is independently predictive of long term mortality in patients with coronary disease. ${ }^{15}$ Moreover, depressed patients with stable coronary disease are much more likely to perceive greater coronary symptom burden and physical limitation compared to non-depressed coronary patients. ${ }^{16}$ Notably, the impaired functional capacity and poor quality of life observed among depressed coronary patients appears to be independent of left ventricular ejection fraction and ischaemic burden as detected through stress echocardiography. ${ }^{16}$

The natural history of depression following ACS is variable. Depressive symptoms may persist for several months following the coronary event or they spontaneously remit. ${ }^{17}{ }^{18}$ For example, among ACS patients with depressive symptoms enrolled in the enhancing recovery in coronary heart disease patients (ENRICHD) trial, the mean score for self reported depressive symptoms declined by $33 \%$ in the six months following hospitalisation in patients randomised to the usual care arm-although some of these patients sought and received out-of-study depression treatment. The mean depression score fell by $49 \%$ among those randomised to depression intervention. ${ }^{12}$ Few studies have simultaneously examined depression and quality of life serially in post-ACS patients. Spertus and colleagues demonstrated that quality of life indicators specific for coronary disease improved or worsened depending on the absence or presence (respectively) of depression symptoms over a three month period. ${ }^{19}$ However, this study was conducted using questionnaires mailed to participants at variable time intervals following their ACS. Lane and colleagues found that depressive symptoms at the time of ACS predicted poorer quality of life at 12 month follow up, however depression status at 12 months was not reported. ${ }^{20}$ Since depression following ACS may remit spontaneously, and because concomitant depression independently worsens quality of life in coronary disease, it is unclear whether a 
depressive episode following ACS predicts poorer quality of life even when the depression remits.

\section{DEPRESSION ADVERSELY AFFECTS QUALITY OF LIFE FOLLOWING MYOCARDIAL INFARCTION}

In the current issue of Heart, DeJonge and colleagues address the complex and changing nature of depression following myocardial infarction and its long term, prospective impact on quality of life. ${ }^{21}$ Patients admitted with myocardial infarction to four hospitals in the Netherlands from 19972000 were recruited for the study. All were enrolled three months postinfarction and their depression status at enrolment was determined through a standardised, diagnostic interview. Questionnaires were also administered at this time to assess self reported depression symptoms and quality of life indicators (both general and specific to coronary disease). At six months postinfarction (three months after study enrolment), patients repeated the self report depression questionnaire. Quality of life was again assessed at 12 months postinfarction, as was depression status via repeat diagnostic interview. Of the 1166 patients admitted with myocardial infarction during the recruitment period, only 421 were ultimately enrolled into the study-a notably low recruitment rate that the authors address nicely. Of these, approximately $25 \%$ were depressed at enrolment, with the majority reporting only mild depressive symptoms (73\%). Among the depressed patients, $17 \%$ had depressive symptoms lasting $>9$ months; most commonly, depression symptoms lasted 3-9 months (46\%), with depression lasting $<3$ months in $37 \%$. The majority of the depressed patients $(60 \%)$ had at least one concomitant anxiety disorder. Depressed myocardial infarction patients were younger, and more likely to be female, smokers, and to have been diagnosed with depression before infarction. There was no difference between the depressed and non-depressed with respect to ejection fraction, Killip class, or prior history of myocardial infarction.

At three months postinfarction, depressed patients admitted to significantly worsened quality of life on every measure, including domains of physical functioning, social functioning, and cardiac related complaints. Most strikingly, depressed patients were more than twice as likely to be completely disabled (35\% v 13\%), and more than three times as likely to be partially disabled $(42 \% v 13 \%)$. At 12 months postinfarction, these findings were essentially unchanged, even when controlling for age, sex, ejection fraction, Killip class, and history of prior infarction or depression. Even when additionally controlling for the severity of social or functional impairment at three months, depressed patients reported lower functional capacity, greater cardiac symptoms and more disability at 12 months, suggesting that depression was predictive of future functional impairment.

To address the prospective impact of depression on quality of life, the authors repeated the analyses, excluding those patients with concurrent depression symptoms at the 12 month assessment $(\mathrm{n}=36(34 \%) ; 18$ patients with longstanding depression and 18 with late onset depression). In this set of subanalyses, patients with any depression symptoms in the year following infarction endorsed significantly poorer physical and social functioning with notably increased risk for disability at 12 months. Cardiac complaints were not more frequent in the absence of concurrent depression, nor were they more frequent when the ejection fraction was analysed continuously (instead of dichotomously). However, depression-and not markers of cardiac status-were much stronger predictors of worse physical and social functioning, increased role limitations, and disability at 12 months. Curiously, the authors did not report the effects of anxiety disorders on 12 month quality of life, though they presented these data in their baseline description of the sample.

\section{DIRECTIONS FOR FUTURE RESEARCH}

What is perhaps most alarming about these findings is the notably increased risk of disability reported among depressed postinfarction patients one year following their infarct. Depression was by far the strongest risk factor for disability at 12 months, even when adjusting for functional impairment at three months and after excluding patients with concurrent depression at the 12 month assessment. Some have advocated treating depression in the ACS population for its own sake. ${ }^{9}$ That is, regardless of the true mechanism(s) that may underlie the association between depression and coronary disease, treating depression is inherently worthwhile. While this may seem obvious, there are some cautionary points to bear in mind:

- To date, there is little evidence to support the suggestion that treating depression will improve quality of life in patients with coronary disease. In fact, in the only randomised trial published to date, depression treatment with sertraline in ACS patients had no beneficial effect on quality of life over placebo (over six months), despite improving depression symptoms. ${ }^{22}$ Of note, however, subgroup analyses in this study revealed that those patients with a history of recurrent depression had modest improvement in quality of life indicators with sertraline treatment.

- Although treating depression in ACS patients seems to make sense, only the minority of patients appear to receive treatment. ${ }^{23}$ In the study by DeJonge et $a l^{21} 74 \%$ of depressed patients reported that they discussed their depression with a healthcare professional, but only $30 \%$ received some form of treatment (13\% treated with antidepressant medication). Similarly, among the depressed patients randomised to usual care in the ENRICHD trial, ${ }^{12}$ only $13 \%$ had been prescribed an antidepressant at six months follow up-although treatment rates increased modestly with longer follow up: $21 \%$ over the full study (mean follow up 29 months).

As investigators move forward in attempting to unravel the intriguing relation between depression and coronary disease, it will be important to include quality of life indicators as outcomes of interest. Indeed, studies should be designed specifically to address improving the functional capacity of depressed ACS patients. It may be premature to assume that simply treating depression symptoms will improve quality of life. As the clinical trials ${ }^{11}{ }^{12}$ have demonstrated, successful treatment of depression does not necessarily modify the risk associated with it. These trials, and the findings of DeJonge et al, ${ }^{21}$ highlight the complex and poorly understood relation between depression and coronary disease. Understanding the fundamental mechanisms linking depression to the increased mortality and morbidity observed in coronary patients will be crucial for the proper design of future trials.

\section{ACKNOWLEDGEMENTS}

The author gratefully acknowledges support from the National Heart, Lung and Blood Institute, National Institutes of Health, Bethesda, MD, USA (HL073599). There are no financial relationships or conflicts of interest to disclose.

\section{REFERENCES}

1 Frasure-Smith N, Lesperance F, Talajic M. Depression and 18-month prognosis after myocardial infarction. Circulation 1995:91:999-1005.

2 Frasure-Smith N, Lesperance F, Gravel G, et al. Social support, depression, and mortality during the first year after myocardial infarction. Circulation 2000;101:1919-24. 
3 Lesperance $F$, Frasure-Smith $N$, Juneau $M$, et al. Depression and 1-year prognosis in unstable angina. Arch Intern Med 2000;160:1354-60.

4 Lesperance $F$, Frasure-Smith N, Talajic $M$, et al. Five-year risk of cardiac mortality in relation to initial severity and one-year changes in depression symptoms after myocardial infarction. Circulation 2002;105:1049-53.

5 Bush DE, Ziegelstein RC, Tayback M, et al. Even minimal symptoms of depression increase mortality risk after acute myocardial infarction. Am J Cardiol 2001;88:337-41.

6 Steeds RP, Channer KS. Depression: the sleeping giant. Eur Heart J 2000;21:427-9.

7 Frasure-Smith N, Lesperance F. Reflections on depression as a cardiac risk factor. Psychosom Med 2005;67(suppl 1):S19-25.

8 Lane D, Ring C, Lip GY, et al. Depression, indirect clinical markers of cardiac disease severity, and mortality following myocardial infarction. Heart 2005.91:531-2.

9 Lane D, Carroll D, Lip GY. Anxiety, depression, and prognosis after myocardial infarction: is there a causal association? J Am Coll Cardiol 2003:42:1808-10.

10 Shimbo D, Davidson KW, Haas DC, et al. Negative impact of depression on outcomes in patients with coronary artery disease: mechanisms, treatment considerations, and future directions. J Thromb Haemost 2005;3:897-908.

11 Glassman AH, O'Connor CM, Califf RM, et al. Sertraline treatment of major depression in patients with acute $\mathrm{MI}$ or unstable angina. JAMA 2002;288:701-9.

12 Berkman LF, Blumenthal J, Burg $M$, et al. Effects of treating depression and low perceived social support on clinical events after myocardial infarction: the enhancing recovery in coronary heart disease patients (ENRICHD) randomized trial. JAMA 2003;289:3106-16.

13 Swenson JR. Quality of life in patients with coronary artery disease and the impact of depression. Curr Psychiatry Rep 2004;6:438-45.
14 Wells KB, Stewart A, Hays RD, et al. The functioning and well-being of depressed patients. Results from the medical outcomes study. JAMA 1989;262:914-9.

15 Spertus JA, Jones $P, M c D o n e l l ~ M$, et al. Health status predicts long-term outcome in outpatients with coronary disease. Circulation 2002;106:43-9.

16 Ruo B, Rumsfeld JS, Hlatky MA, et al. Depressive symptoms and health-related quality of life: the heart and soul study. JAMA 2003;290:215-21.

17 Schleifer SJ, Macari-Hinson MM, Coyle DA, et al. The nature and course of depression following myocardial infarction. Arch Intern Med 1989;149:1785-9.

18 Lesperance F, Frasure-Smith N, Talajic M. Major depression before and after myocardial infarction: its nature and consequences. Psychosom Med 1996;58:99-110

19 Spertus JA, McDonell M, Woodman CL, et al. Association between depression and worse disease-specific functional status in outpatients with coronary artery disease. Am Heart J 2000;140:105-10.

20 Lane D, Carroll D, Ring C, et al. Mortality and quality of life 12 months after myocardial infarction: effects of depression and anxiety. Psychosom Med 2001;63:221-30.

21 de Jonge $\mathbf{P}$, Spiikerman TA, van den Brink RHS, et al. Depression after myocardial infarction is a risk factor for declining health related quality of life and increased disability and cardiac complaints at 12 months. Heart 2006;92:32-9

22 Swenson JR, O'Connor CM, Barton D, et al. Influence of depression and effect of treatment with sertraline on quality of life after hospitalization for acute coronary syndrome. Am J Cardiol 2003;92:1271-6.

23 Ziegelstein RC, Kim SY, Kao D, et al. Can doctors and nurses recognize depression in patients hospitalized with an acute myocardial infarction in the absence of formal screening? Psychosom Med 2005;67:393-7.

\section{IMAGES IN CARDIOLOGY}

\section{An unexpected complication during right jugular vein cannulation in a young adult}

$\mathrm{T}$ he figure presents a complete atrioventricular (AV) block which developed in a 35 year old woman hospitalised in our intensive care unit because of septic shock. She had been first hospitalised in the gastroenterology department because of an upper gastrointestinal tract haemorrhage caused by oesophageal varices in the context of hepatic cirrhosis of unknown cause. The AV block developed when we attempted to insert a right jugular vein catheter several days later. During the insertion of the guidewire the patient developed a brief run of atrial tachycardia followed by a period of complete atrioventricular block. Her arterial pressure fell dramatically. The guidewire was partially withdrawn and her cardiac rhythm and pressure were restored to normal.

Our patient did not present any cardiac abnormality. The admission ECG demonstrated a sinus tachycardia ( 136 beats/ min) and a P-R interval of $0.12 \mathrm{~s}$ while cardiac enzyme measurements performed the days that followed her admission were normal. After the event, factors that could explain the block such as acidosis, electrolyte disturbances, endocarditis, large ascitic fluid quantity, and medications (for example, $\beta$ blockers) were ruled out.

Arrhythmias during central venous cannulation are more prone to occur with the insertion of a pulmonary arterial catheter, especially if a pre-existing left bundle branch block (LBBB) is present. In literature, the induction of a complete heart block during the insertion of a central venous catheter has been described in two older patients with a pre-existing LBBB. The most probable explanation in our patient is a transient injury to the distal AV node caused by the overinsertion of the guide wire. Care must be taken when inserting guidewires under continuous monitoring in a distance less than $16-18 \mathrm{~cm}$, since life threatening arrhythmias can occur even in young patients without cardiac abnormalities.

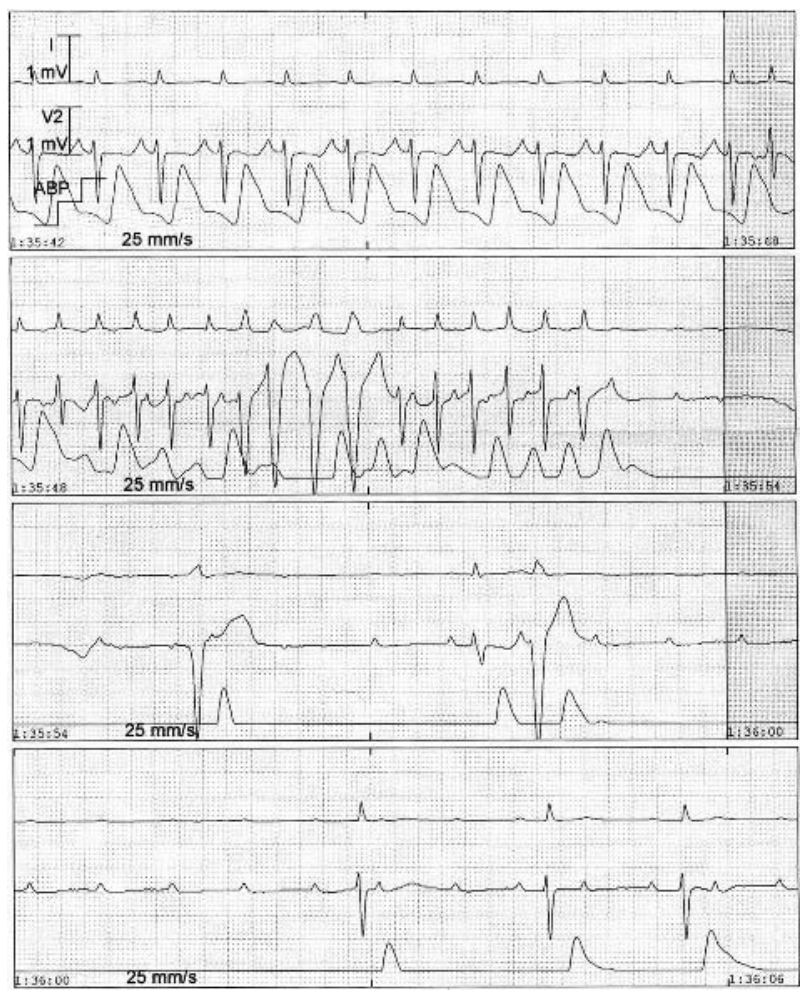

E Paramythiotou I Poularas A Kalogeromitros V Krikos

N Katsarelis

A Karabinis lparamyth61@hotmail.com 